\title{
Atomic-Scale Observation of Oxygen Vacancy-Induced Step Reconstruction in WO3
}

\author{
Meng, Jie; Lan, Zhenyun; Castelli, Ivano Eligio; Zheng, Kaibo
}

Published in:

Journal of Physical Chemistry C

Link to article, DOI:

10.1021/acs.jpcc.1c01577

Publication date:

2021

Document Version

Publisher's PDF, also known as Version of record

Link back to DTU Orbit

Citation (APA):

Meng, J., Lan, Z., Castelli, I. E., \& Zheng, K. (2021). Atomic-Scale Observation of Oxygen Vacancy-Induced Step Reconstruction in $\mathrm{WO}_{3}$. Journal of Physical Chemistry C, 125(15), 8456-8460.

https://doi.org/10.1021/acs.jpcc.1c01577

\section{General rights}

Copyright and moral rights for the publications made accessible in the public portal are retained by the authors and/or other copyright owners and it is a condition of accessing publications that users recognise and abide by the legal requirements associated with these rights.

- Users may download and print one copy of any publication from the public portal for the purpose of private study or research.

- You may not further distribute the material or use it for any profit-making activity or commercial gain

- You may freely distribute the URL identifying the publication in the public portal

If you believe that this document breaches copyright please contact us providing details, and we will remove access to the work immediately and investigate your claim. 


\title{
Atomic-Scale Observation of Oxygen Vacancy-Induced Step Reconstruction in $\mathrm{WO}_{3}$
}

\author{
Jie Meng," Zhenyun Lan," Ivano E. Castelli, and Kaibo Zheng*
}

Cite This: J. Phys. Chem. C 2021, 125, 8456-8460

Read Online

ABSTRACT: As the most common type of defects in $\mathrm{WO}_{3}$, surface oxygen deficiencies have been widely investigated in terms of their structure as well as physicochemical properties. In general, oxygen vacancy on the surface may lead to the reconstruction of the surface structure due to the need for surface energy minimization, especially for the area with high oxygen vacancy concentration. However, such a phenomenon has not been directly demonstrated or observed from the experiment. In the paper, we observed such surface reconstruction in the step area or high-index facets in $\mathrm{WO}_{3}$ using the spherical aberration-corrected scanning transmission electron microscope (Cs-STEM) with atomic-scale resolution. This surface reconstruction results in a structure of 5-fold symmetry pentagonal columns, which is first reported. According to the DFT calculation, the formation energy of oxygen vacancy in the step edge is much lower than the smooth surface. Furthermore, the formation energy of oxygen vacancy in the higher index (110) facet is lower than the low index (100) and (010) facets. Our experimental results support these findings, i.e., that the reconstruction is pronounced in the step or high-index facets with a high concentration of oxygen vacancy. Therefore, the high concentration of oxygen vacancy in the step or high-index facet area contributes to the formation of pentagonal column structures. This work could provide a novel insight into the correlation between intrinsic defect and surface structure formation in these materials.

\section{INTRODUCTION}

Tungsten oxides $\left(\mathrm{WO}_{3}\right)$ have attracted great interest in various applications including sensors, ${ }^{1}$ (photo) catalysts, ${ }^{2-4}$ electrochromic/optochromic devices, etc., ${ }^{3,5}$ Oxygen vacancies are the most observed structural defects in $\mathrm{WO}_{3}{ }^{7}$ In general, oxygen vacancies serve as shallow donors in $\mathrm{WO}_{3}$ that can provide electron carrier concentration and promote n-type conductivity. ${ }^{8}$ They can also induce the formation of the polaron that modifies the local electronic structures.

The structure and properties of oxygen vacancies in the bulk $\mathrm{WO}_{3}$ have been extensively studied., ${ }^{8,10,11}$ However, the situation may be entirely different when such vacancies reside at the surface of the material. ${ }^{12}$ Since the surface is sensitive to the surrounding environment, ${ }^{13}$ the surface atoms will tend to be displaced from their original sites determined by the crystal symmetry of the bulk in order to minimize the surface energy. ${ }^{14}$ This so-called surface reconstruction occurs when the symmetry of the crystal is broken from the bulk to the surface. ${ }^{15}$ Such structural reconstructed surfaces usually induce unconventional physical and chemical properties compared to the bulk materials. ${ }^{16-19}$ As the existence of oxygen vacancy further breaks the local lattice symmetry, we can expect an enhanced surface reconstruction. ${ }^{20}$ However, this possible oxygen vacancy induced surface reconstruction has never been clearly visualized experimentally in $\mathrm{WO}_{3}$. The emerging Cscorrected transmission electron microscopy (Cs-STEM) with ultimate sub-Ångström resolution enables the monitoring of such surface reconstruction at atomic scale. ${ }^{21}$
In this work, we characterized the surface structure of $\mathrm{WO}_{3}$ with a special focus on the step area or high-index facets using the spherical aberration-corrected scanning transmission electron microscope (Cs-STEM) with atomic-scale resolution. We observe the formation of characteristic pentagonal column structures in those areas for the first time. Density Functional Theory (DFT) calculations indicate the low formation energy of oxygen vacancy in all those step structures or high-index facets. Therefore, we concluded the critical role of oxygen vacancy in displacing the neighboring $\mathrm{W}$ atoms column position and consequently inducing the surface reconstruction.

\section{RESULTS AND DISCUSSION}

The $\mathrm{WO}_{3}$ nanocrystals were synthesized by a solid-state method and can be well indexed into a monoclinic phase from the XRD pattern (space group: $P 2_{1} / n$ ) in Figure S1. No impurities were found from XRD characterization. The ideal crystal structure of monoclinic $\mathrm{WO}_{3}$ is identical to the $\mathrm{ReO}_{3}$ type or $\mathrm{ABO}_{3}$ perovskite structure in the absence of an $\mathrm{A}$ cation, i.e., a three-dimensional network was formed by tilted

Received: February 21, 2021

Revised: $\quad$ March 23, 2021

Published: April 7, 2021 
corner-sharing $\mathrm{WO}_{6}$ octahedra as illustrated in Figure $1 \mathrm{a}^{22}$ The HAADF-STEM image of $\mathrm{WO}_{3}$ nanocrystals viewed along
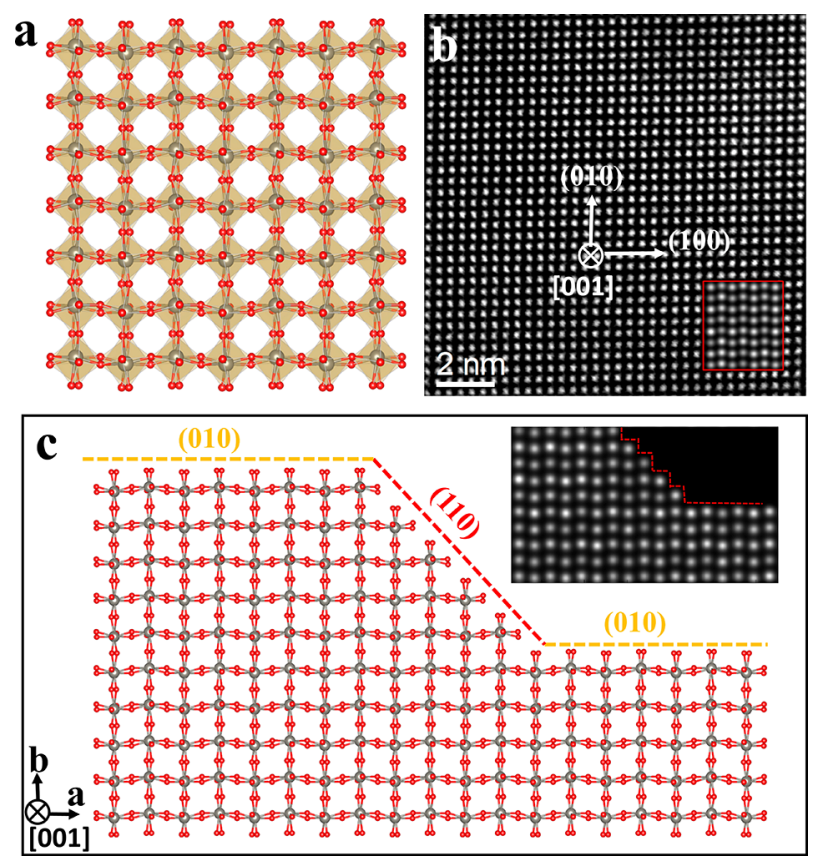

Figure 1. Atomic structure of $\mathrm{WO}_{3}$. (a) Atomic model of $\mathrm{WO}_{3}$ under the [001] zone axis (W: gray atoms and O: red atoms). (b) Simulated (inset) and experimental HAADF-STEM image of the bulk part for $\mathrm{WO}_{3}$ nanocrystals with atomic scale under the [001] zone axis. (c) The surface atomic structures and corresponding HAADF-STEM simulation of $\mathrm{WO}_{3}$ with (010) and (110) exposed facets.

the [001] zone axis is shown in Figure $1 \mathrm{~b}$ with the simulation image presented as an inset in Figure $1 \mathrm{~b}$. The details of the HAADF-STEM image simulation are described in the experimental section of the Supporting Information. Since the image contrast in HAADF-STEM is roughly proportional to $Z^{1.6-2.0}$ ( $Z$ : atomic number), the bright dots in Figure 1 indicate the overlapped $\mathrm{W}-\mathrm{O}$ atomic columns. ${ }^{23}$ Oxygen columns are not visible in the image due to the small scattering intensity for the light element. The experimental and simulated HAADF-STEM images further confirm the monoclinic phase of the sample. Although there are the tilted corner-sharing $\mathrm{WO}_{6}$ octahedra in monoclinic $\mathrm{WO}_{3}$, anisotropy among three low-index facets, such as (100), (010), and (001), is not obvious. Therefore, in this work, we target (010), (100), and (110). The ideal atomic structure model with the absence of any defects and corresponding ordered arrangement of $\mathrm{W}-\mathrm{O}$ atomic columns of $\mathrm{WO}_{3}$ with (010) and (110) facets should be identical as presented in Figure 1c. The higher index facets, such as (110) facets, normally will grow as steps compared to the lower index (100) facet shown in Figure 2.

Figure 2a shows the morphology (inset image) and surface structure of $\mathrm{WO}_{3}$ nanocrystals. The (110) and (010) exposed surfaces along the [001] zone axis direction are marked by red and yellow dashed lines, respectively (Figure $2 \mathrm{~b}$ ). The surface usually will be arranged as the $\mathrm{ReO}_{3}$ type or $\mathrm{ABO}_{3}$ perovskite structure if there is no defect in the surface as shown in Figure 1c. However, we can clearly observe pentagonal columns with 5 -fold symmetry exist in the (110) surface as shown in Figure 2b (marked by the red dashed lines). Interestingly, such surface reconstruction is only found in the (110) surface but not in the (010) surface. This pentagonal column structure in tungsten oxides $\left(\mathrm{WO}_{3-x}\right)$ also exists in $\mathrm{W}_{18} \mathrm{O}_{49}$ or $\mathrm{WO}_{2.7}$ when there are abundant oxygen vacancies as illustrated in Figure $2 \mathrm{e}^{24}$ As the number of oxygen vacancies increases, $\mathrm{WO}_{6}$ octahedra in the crystal structure change position from cornerto edge-sharing split by crystallographic shear facets. Nonstoichiometric tungsten with an oxygen deficiency, $\mathrm{WO}_{x}(x=$ 2.6-3), has various well-defined suboxides known as Magneli

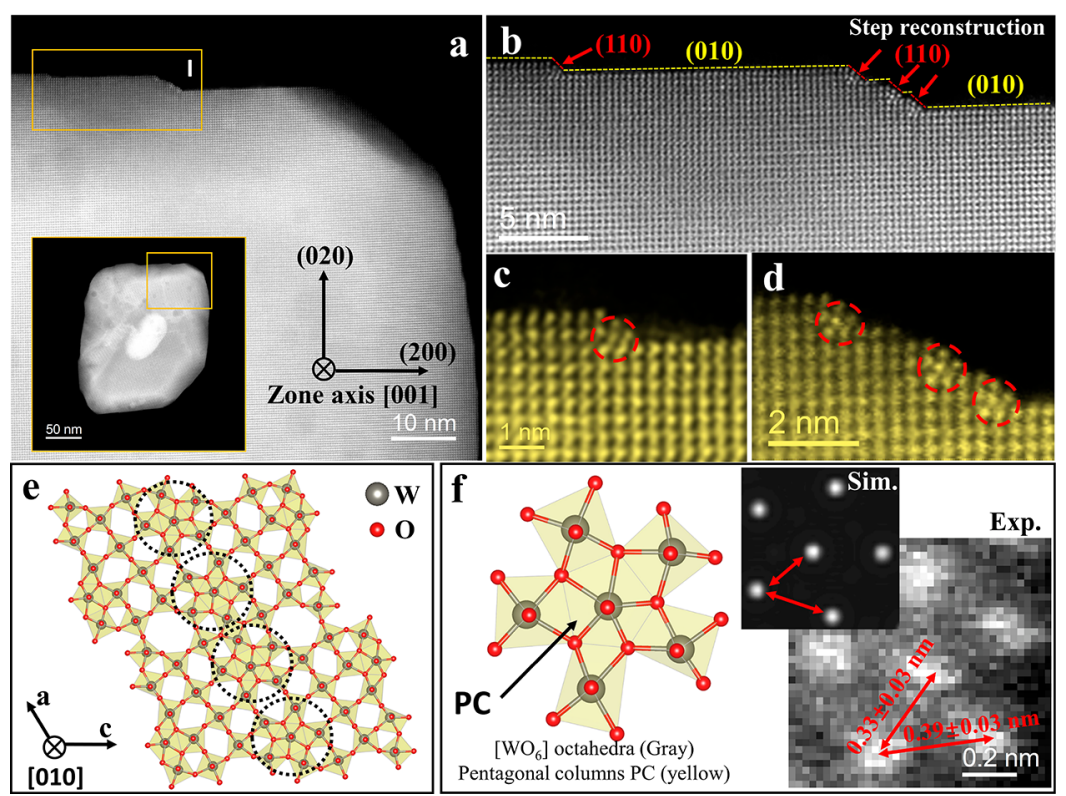

Figure 2. Atomic-resolution STEM-HAADF images of $\mathrm{WO}_{3}$ nanocrystals. (a) Morphology and surface structure of WO $\mathrm{W}_{3}$. Area $\mathrm{I}$ is the step position. (b) Atomic structure of the $\mathrm{WO}_{3}$ surface with (010) (yellow dashed lines) and (110) (red dashed lines). (c,d) Atomic structures of step reconstruction. Parts $\mathrm{c}$ and $\mathrm{d}$ are from the left and right (110) facet areas in part b, respectively. (e) Atomic structures of $\mathrm{W}_{18} \mathrm{O}_{49}$ with $\left[\mathrm{WO}_{6}\right]$ octahedra and pentagonal columns (PC) (marked by the black dashed circles). (f) Crystal structure of the pentagonal columns. The right parts are the corresponding HAADF-STEM simulation and experimental images. The errors come from the pixel size. 


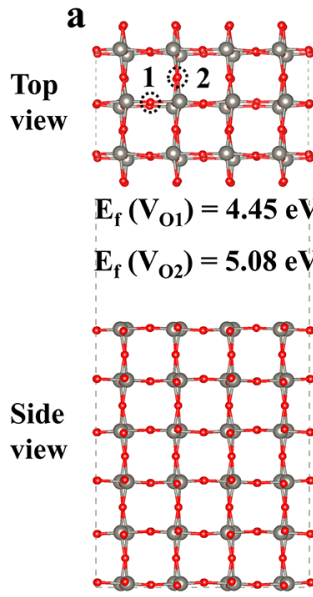

(100) b

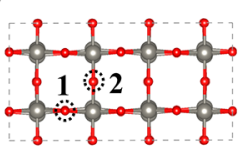

$\mathrm{E}_{\mathrm{f}}\left(\mathrm{V}_{\mathrm{O} 1}\right)=5.12 \mathrm{eV}$

$\mathrm{E}_{\mathrm{f}}\left(\mathrm{V}_{\mathrm{O} 2}\right)=4.88 \mathrm{eV}$

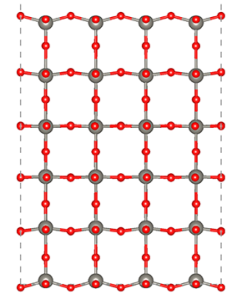

(010) c

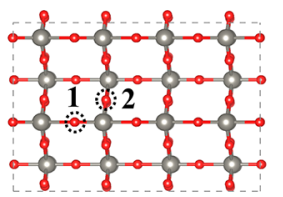

$\mathrm{E}_{\mathrm{f}}\left(\mathrm{V}_{\mathrm{O} 1}\right)=-4.45 \mathrm{eV}$

$E_{f}\left(V_{02}\right)=-4.95 \mathrm{eV}$

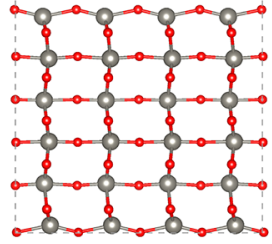

(110) d Sites $\mathrm{E}_{\mathrm{f}}\left(\mathrm{V}_{\mathrm{o}}\right)$

$1 \quad 4.30 \mathrm{eV}$

$2 \quad-0.96 \mathrm{eV}$

$3 \quad 0.19 \mathrm{eV}$

$4-\mathbf{0 . 5 2} \mathrm{eV}$

$0.28 \mathrm{eV}$

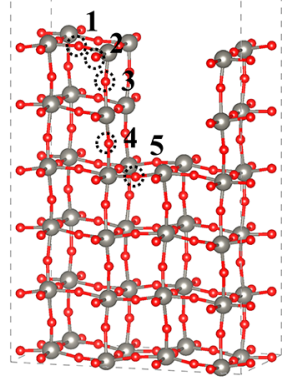

Step structures

Figure 3. Calculated formation energies of oxygen vacancy. The top view and side view of (a) the (100) surface, (b) (010) surface, and (c) (110) surface structure. (d) The formation energies of oxygen vacancy in step structures. The $\mathrm{W}$ and $\mathrm{O}$ atoms are marked by gray and red colors, respectively. The positions of oxygen vacancy are labeled by the black dashed circles.
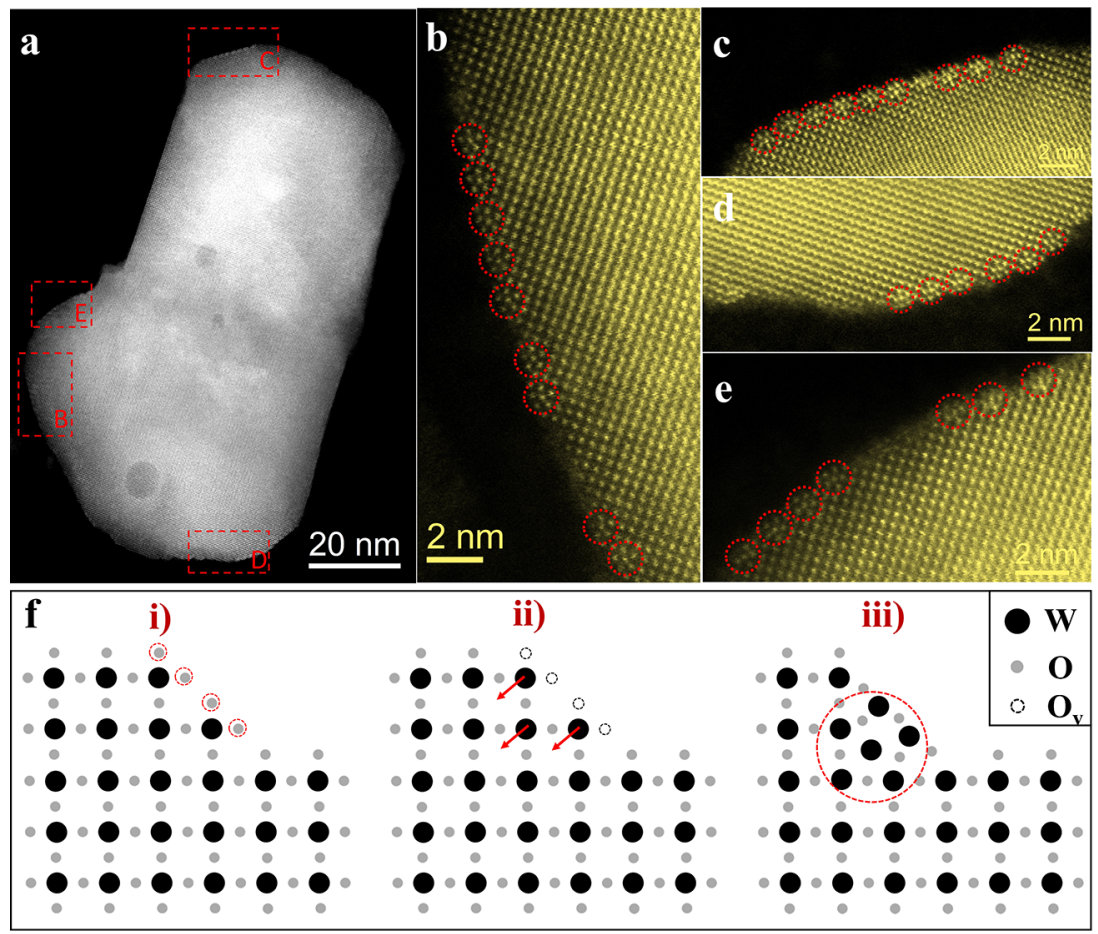

Figure 4. Atomic-resolution STEM-HAADF images of $\mathrm{WO}_{3}$ nanocrystals with more oxygen vacancies. (a) Morphology of WO $\mathrm{W}_{3}$. (b) Surface atomic structure of $\mathrm{WO}_{3}$ in area B. (c) Surface atomic structure of $\mathrm{WO}_{3}$ in area C. (d) Surface atomic structure of $\mathrm{WO}_{3}$ in area D. (e) Surface atomic structure of $\mathrm{WO}_{3}$ in area $\mathrm{E}$. The reconstruction areas are marked by red dashed circles. To make the HAADF-STEM images more clear, the pictures are marked by the color yellow, while it would not change the structure information. (f) The schematic images of surface reconstruction: (i) part of the oxygen at the edge will be easily lost, (ii) the formation of $\mathrm{O}_{\mathrm{v}}$ and $\mathrm{W}$ atom arrangement, and (iii) surface reconstruction. The positions for oxygen vacancy representing oxygen will be easily lost in these areas and do not indicate all the oxygen will be removed.

phases. $^{25}$ Among all suboxides, such as $\mathrm{W}_{32} \mathrm{O}_{84}\left(\mathrm{WO}_{2.625}\right)$, $\mathrm{W}_{3} \mathrm{O}_{8}\left(\mathrm{WO}_{2.667}\right), \mathrm{W}_{18} \mathrm{O}_{49}\left(\mathrm{WO}_{2.72}\right), \mathrm{W}_{17} \mathrm{O}_{47}\left(\mathrm{WO}_{2.765}\right), \mathrm{W}_{5} \mathrm{O}_{14}$ $\left(\mathrm{WO}_{2.8}\right), \mathrm{W}_{20} \mathrm{O}_{58}\left(\mathrm{WO}_{2.9}\right), \mathrm{W}_{2} \mathrm{O}_{5}, \mathrm{WO}_{2}$, and $\mathrm{W}_{25} \mathrm{O}_{73}\left(\mathrm{WO}_{2.92}\right)$, nonstoichiometric $\mathrm{W}_{18} \mathrm{O}_{49}$ is the most commonly observed crystal structure. ${ }^{2}$ It contains both octahedral and pentagonal coordinations of the metal atoms by oxygen. The distance from centered $\mathrm{W}$ columns to adjacent $\mathrm{W}$ columns is around 0.33 $\mathrm{nm}$. The distance between two neighboring $\mathrm{W}$ columns is about $0.39 \mathrm{~nm}$. These results agree well with the simulation or the standard $\mathrm{W}-\mathrm{W}$ distance (Figure 2e). That is to say, this 5 fold structure agrees well with pentagonal columns.

Oxygen and the presence of oxygen vacancies thus play an important role in the surface reconstruction. To further evaluate their influence, we calculate the formation energies of oxygen vacancy using DFT. We started by calculating the formation energy at various surfaces (i.e., (100), (010), and $(110))$. Due to the existence of anisotropy in $\mathrm{WO}_{3}$, two representative sites were chosen for each surface. As shown in Figures $3 a-3 c$, the formation energy of oxygen vacancy in the 
(110) facet is the lowest compared with (100) and (010) facets. Moreover, the formation energies in (110) are negative, indicating the spontaneous formation of oxygen vacancy in this facet. There are three main growth modes accepted: the Volmer-Weber mode (island growth), the Frank-van der Merwe mode (layer-by-layer growth), and the StranskiKrastanov mode (mixed growth). ${ }^{26}$ These growth modes typically will contribute to the formation of step structures, which are known as high-index surfaces. In the next step, we reproduce the step structure at the (110) surface and simulate the formation of oxygen vacancy at five representative sites 15 within the step as shown in Figure 3d. The oxygen vacancy at the sites closer to the step edges (i.e., sites 2-4) exhibits lower formation energies with $-0.96,-0.52$, and $0.19 \mathrm{eV}$, respectively. Such calculation indicates the oxygen vacancies are easier to generate at the step area, which could be expected as the driving force of the above-mentioned surface reconstruction observed at the same place. In order to further support our conclusion that the 5-fold symmetry pentagonal column formation due occurs at the place with the existence of oxygen vacancy, we conducted the EDS characterization. As shown in Figure S3 and Table S1 (SI), the atomic ratio in the edge area is lower than the smooth surface, indicating that the edge area exhibits higher oxygen vacancy concentration.

In order to further verify our assumption, we manually increase oxygen vacancy concentration in the $\mathrm{WO}_{3}$ by modifying the solid-state synthesis method under $\mathrm{Ar}$ circulation environment. The as-obtained Vo-rich $\mathrm{WO}_{3}$ can be well indexed into the monoclinic phase without any other impurities. The HAADF STEM images of the Vo-rich $\mathrm{WO}_{3}$ nanocrystals are shown in Figure $4 a$ and Figure $4 b-e$ with larger magnification. It is obvious that Vo-rich $\mathrm{WO}_{3}$ exhibits many more pentagonal column structures on the surface. ${ }^{27}$ The increased pentagonal columns in Vo-rich $\mathrm{WO}_{3}$ manifest the critical role of oxygen vacancy in promoting the surface reconstruction to pentagonal column structures. The underlying mechanism for the surface reconstruction is proposed in Figure $4 \mathrm{f}$. Some of the oxygen atoms around the $\mathrm{W}$ atoms at the step area are easily lost forming oxygen vacancies (i). The existence of oxygen vacancy breaks the local symmetry and lowers the structure of $\mathrm{WO}_{3}$. Therefore, $\mathrm{W}$ atoms in the edge positions will squeeze into the void area and coordinate with neighboring atoms (ii) and finally form the pentagonal column structures (iii) in order to minimize the surface energy and stabilize the structures.

\section{CONCLUSIONS}

In conclusion, through the spherical aberration-corrected scanning transmission electron microscope (Cs-STEM) with atomic-scale resolution, we for the first time observed surface reconstruction in the step area or high-index facets of $\mathrm{WO}_{3}$ into 5-fold symmetry pentagonal column structures. DFT calculation indicates those areas in $\mathrm{WO}_{3}$ possess much low oxygen vacancy formation energy. Besides, we confirmed the enhancement of such surface reconstruction with the increase of oxygen vacancy concentration. This demonstrated that the oxygen vacancies promote or facilitate surface reconstruction. This work provides a new insight into the deficiency-related surface reaction in these materials. It also indicates the intrinsic oxygen vacancy may be utilized as a robust tool for the engineering of the surface structure in $\mathrm{WO}_{3}$. Meanwhile, more direct evidence for in situ observed surface reconstruction environmental TEM under different $\mathrm{O}_{2}$ pressures can be further explored in the future.

\section{ASSOCIATED CONTENT}

\section{Supporting Information}

The Supporting Information is available free of charge at https://pubs.acs.org/doi/10.1021/acs.jpcc.1c01577.

Experimental section, DFT calculation, XRD spectra of $\mathrm{WO}_{3}$ with low and high oxygen vacancy (PDF)

\section{AUTHOR INFORMATION}

\section{Corresponding Author}

Kaibo Zheng - Department of Chemistry, Technical University of Denmark, DK-2800 Kongens Lyngby, Denmark; Chemical Physics and NanoLund, Lund University, 22100 Lund, Sweden; 이이. orcido000-00027236-1070; Email: kzheng@kemi.dtu.dk, kaibo.zheng@ chemphys.lu.se

\section{Authors}

Jie Meng - Department of Chemistry, Technical University of Denmark, DK-2800 Kongens Lyngby, Denmark; (1) orcid.org/0000-0002-3813-5221

Zhenyun Lan - Department of Energy Conversion and Storage, Technical University of Denmark, DK-2800 Kongens Lyngby, Denmark; ○ orcid.org/0000-0001-7943-5936

Ivano E. Castelli - Department of Energy Conversion and Storage, Technical University of Denmark, DK-2800 Kongens Lyngby, Denmark; 이이. orcid.org/0000-0001-5880-5045

Complete contact information is available at: https://pubs.acs.org/10.1021/acs.jpcc.1c01577

\section{Author Contributions}

"J.M. and Z.L. contributed equally to this work.

\section{Notes}

The authors declare no competing financial interest.

\section{ACKNOWLEDGMENTS}

This work was supported by the Independent Research Fund Denmark-Sapere Aude starting grant (no. 7026-00037A), a Swedish Research Council VR starting grant (no. 201705337), the Crafood Foundation (no. 20200522), a Swedish Energy Agency research grant, and the Research Fund for International Young Scientists from NSFC, China (no. 21950410515) (K.Z.). J.M. and Z.L. acknowledge financial support from the China Scholarship Council.

\section{REFERENCES}

(1) Dong, C.; Zhao, R.; Yao, L.; Ran, Y.; Zhang, X.; Wang, Y. A Review on $\mathrm{WO}_{3}$ Based Gas Sensors: Morphology Control and Enhanced Sensing Properties. J. Alloys Compd. 2020, 820, 153194.

(2) Shinde, P. A.; Jun, S. C. Review on Recent Progress in the Development of Tungsten Oxide Based Electrodes for Electrochemical Energy Storage. ChemSusChem 2020, 13, 11-38.

(3) Deb, S. K. Opportunities and Challenges in Science and Technology of $\mathrm{WO}_{3}$ for Electrochromic and Related Applications. Sol. Energy Mater. Sol. Cells 2008, 92, 245-258.

(4) Wang, Z.; Wang, L. Photoelectrode for Water Splitting: Materials, Fabrication and Characterization. Sci. China Mater. 2018, 61, 806-821.

(5) Baeck, S.-H.; Choi, K.-S.; Jaramillo, T. F.; Stucky, G. D.; McFarland, E. W. Enhancement of Photocatalytic and Electrochromic 
Properties of Electrochemically Fabricated Mesoporous $\mathrm{WO}_{3}$ Thin Films. Adv. Mater. 2003, 15, 1269-1273.

(6) Wang, S.; Fan, W.; Liu, Z.; Yu, A.; Jiang, X. Advances on Tungsten Oxide Based Photochromic Materials: Strategies to Improve Their Photochromic Properties. J. Mater. Chem. C 2018, 6, 191-212.

(7) Gerosa, M.; Di Valentin, C.; Onida, G.; Bottani, C. E.; Pacchioni, G. Anisotropic Effects of Oxygen Vacancies on Electrochromic Properties and Conductivity of $\gamma$-Monoclinic $\mathrm{WO}_{3}$. J. Phys. Chem. C 2016, 120, 11716-11726.

(8) Berak, J. M.; Sienko, M. J. Effect of Oxygen-Deficiency on Electrical Transport Properties of Tungsten Trioxide Crystals. J. Solid State Chem. 1970, 2, 109-133.

(9) Wang, W.; Janotti, A.; Van de Walle, C. G. Role of Oxygen Vacancies in Crystalline $\mathrm{WO}_{3}$. J. Mater. Chem. C 2016, 4, 6641-6648.

(10) Corby, S.; Francàs, L.; Kafizas, A.; Durrant, J. R. Determining the Role of Oxygen Vacancies in the Photoelectrocatalytic Performance of $\mathrm{WO}_{3}$ for Water Oxidation. Chem. Sci. 2020, 11, 2907-2914.

(11) Lambert-Mauriat, C.; Oison, V. Density-Functional Study of Oxygen Vacancies in Monoclinic Tungsten Oxide. J. Phys.: Condens. Matter 2006, 18, 7361-7371.

(12) Zhuang, G.; Chen, Y.; Zhuang, Z.; Yu, Y.; Yu, J. Oxygen Vacancies in Metal Oxides: Recent Progress towards Advanced Catalyst Design. Sci. China Mater. 2020, 63, 2089-2118.

(13) Newell, D. T.; Harrison, A.; Silly, F.; Castell, M. R. $\mathrm{SrTiO}_{3}$ (001)- $(\sqrt{5} \times \sqrt{5})-R 26.6^{\circ}$ reconstruction: A surface. Phys. Rev. B: Condens. Matter Mater. Phys. 2007, 75, 205429.

(14) Xu, W.; Bowes, P. C.; Grimley, E. D.; Irving, D. L.; LeBeau, J. M. In-Situ Real-Space Imaging of Single Crystal Surface Reconstructions via Electron Microscopy. Appl. Phys. Lett. 2016, 109, 201601.

(15) Chadi, D. J. Atomic and Electronic Structures of Reconstructed Si(100) Surfaces. Phys. Rev. Lett. 1979, 43, 43-47.

(16) Ignatans, R.; Mallia, G.; Ahmad, E. A.; Spillane, L.; Stoerzinger, K. A.; Shao-Horn, Y.; Harrison, N. M.; Tileli, V. The Effect of Surface Reconstruction on the Oxygen Reduction Reaction Properties of $\mathrm{LaMnO}_{3}$. J. Phys. Chem. C 2019, 123, 11621-11627.

(17) Duan, Y.; Sun, S.; Sun, Y.; Xi, S.; Chi, X.; Zhang, Q.; Ren, X.; Wang, J.; Ong, S. J. H.; Du, Y.; et al. Mastering Surface Reconstruction of Metastable Spinel Oxides for Better Water Oxidation. Adv. Mater. 2019, 31, 1807898.

(18) Liu, X.; Yao, G.; Cheng, X.; Xu, J.; Cai, X.; Hu, W.; Xu, W. W.; Zhang, C.; Zhu, Y. Cd-Driven Surface Reconstruction and Photodynamics in Gold Nanoclusters. Chem. Sci. 2021, 12, 3290.

(19) Huang, J.; Yu, Y.; Zhu, J.; Yu, R. Atomic Layer Reversal on $\mathrm{CeO}_{2}$ (100) Surface. Science China Materials 2017, 60, 903-908.

(20) Sarkar, A.; Khan, G. G. The Formation and Detection Techniques of Oxygen Vacancies in Titanium Oxide-Based Nanostructures. Nanoscale 2019, 11, 3414-3444.

(21) Urban, K. W. Studying Atomic Structures by AberrationCorrected Transmission Electron Microscopy. Science 2008, 321, 506-510.

(22) Quan, H.; Gao, Y.; Wang, W. Tungsten Oxide-Based Visible Light-Driven Photocatalysts: Crystal and Electronic Structures and Strategies for Photocatalytic Efficiency Enhancement. Inorg. Chem. Front. 2020, 7, 817-838.

(23) Sakaguchi, N.; Yamaki, F.; Saito, G.; Kunisada, Y. Estimating the Dopant Distribution in Ca-Doped $\alpha$-SiAlON: Statistical HAADFSTEM Analysis and Large-Scale Atomic Modeling. Microscopy 2016, 65, 400-406.

(24) Viswanathan, K.; Brandt, K.; Salje, E. Crystal Structure and Charge Carrier Concentration of $\mathrm{W}_{18} \mathrm{O}_{49}$. J. Solid State Chem. 1981, $36,45-51$.

(25) Migas, D. B.; Shaposhnikov, V. L.; Borisenko, V. E. Tungsten Oxides. II. The Metallic Nature of Magnéli Phases. J. Appl. Phys. 2010, 108, 093714.

(26) Levi, A. C.; Kotrla, M. Theory and Simulation of Crystal Growth. J. Phys.: Condens. Matter 1997, 9, 299-344.

(27) Sun, Z.; Huo, R.; Choi, C.; Hong, S.; Wu, T.-S.; Qiu, J.; Yan, C.; Han, Z.; Liu, Y.; Soo, Y.-L.; et al. Oxygen Vacancy Enables
Electrochemical $\mathrm{N}_{2}$ Fixation over $\mathrm{WO}_{3}$ with Tailored Structure. Nano Energy 2019, 62, 869-875. 